\title{
A Novel Olfactory Displays' Scent Dispersing Module
}

\author{
Saad Hameed Abid ${ }^{1,2}$, Zhiyong $\mathrm{Li}^{1}$, Renfa $\mathrm{Li}^{1}$ and Jumana Waleed ${ }^{3}$ \\ ${ }^{1}$ College of Computer Sciences and Electronic Engineering, \\ Hunan University, Changsha, Hunan 410082, China \\ saad@hnu.edu.cn, zhiyong.li@hnu.edu.cn, \\ lirenfa@hnu.edu.cn \\ ${ }^{2}$ AL-Mansour University College, Al-Andalus square, Baghdad, Iraq \\ ${ }^{3}$ School of information science and engineering, Central South University, \\ Changsha, 410083, China \\ jumana@csu.edu.cn
}

\begin{abstract}
Olfactory display devices have become a recent demand and trend in human computer interaction, providing a new member in the human computer interaction peripheral devices family. These devices enrich the olfactory sense for the user stimulating him/her by means of scent stimuli in correspondence to an object or an event shown on the visual display. Some researches have been done to cover the design, usage and delivery for the scent to the users, other works covered some analysis on the scent categorization and grouping. In this paper a focus is on the controlled atomization of a liquid based scent material while guaranteeing: speed, intensity, durability, compactness and scalability, these important factors are crucial to build a commercial grade olfactory displays containing a universal, interchangeable and versatile but yet durable scent disparity component to facilitate the realization of olfactory display devices.
\end{abstract}

Keywords: Scent delivery element, Olfactory displays, scent diffusion, atomization

\section{Introduction}

While the user interacting with the traditional display that stimulating his human visual system (HVS), he/she might encounter a "Scent object" or a "Scent Related Event", both scent objects and scent related events encountering are expressed as a situation where a user should interpret using his/her human olfactory system HOS stimulating his/her smell related brain cells to obtain more immersive experience with the computer. The smell object could be an apple displayed on the screen or maybe a basket filled with apples, to distinguish these two objects scent intensity should be controlled during the delivery event, obviously the single apple requires the system to deliver less scent intensity than a basket of apples. For the same apple the amount of distance between the apple and the viewer is also affect the intensity of the scent, the closer the object the higher the intensity. Furthermore in virtual environment it is essential to intercept all users' actual perception with injected virtual data and this olfactory system still a virgin field of research. To accomplish that we need an olfactory display that is capable of successfully delivering the required scented information accurately and immediately to cope with the rapidly changing data that the user is exploring.

\section{Related Works}

Many researches have been done in the field of olfactory displays, several approaches and technologies were applied to disperse the scented data to the targeted user. In [1] and [2] a projection device were proposed to eject the scent 
based on nose tracking feature, the ejection is done using a proposed air cannon that contracts upon delivery. The scent in delivered inside the cannon and ejected afterwards, while in [3-6] the same olfactory display were used to disperse the scented material towards the user, it uses solenoids valves and an air compressor to create an air flow. The concept is based on bubbling the air into the liquid scented material to create an evaporated scent mixed with air (air born scent) and controlling the flow of that air/scent mixture among other types of scents produced in the same way to be directed to the user's nose. Furthermore in [7] and [8] another approach were applied using an electro-osmotic micro pump to deliver a predefined amount of scented material to be dispersed using and SAW (surface acoustic wave) device that is capable of producing a vapor rapidly, other techniques such as [9] using a solid (gel) scent material and a peltier module, the module produces heat to transform the gel material to vapor and deliver it to the user. In the previously mentioned techniques the olfactory display device is basically a desktop device connected to a personal computer and other supportive devices such as high voltage supply, air pump and frequency generators etc. while in [10-12] the olfactory display were worn by the user and the scent ejection point is placed near the user's nose, this approach is suitable for virtual reality application when the user is need to be mobile rather than sitting at a desk. The mentioned techniques solved some problems in terms of scent delver but still have limitations in terms of intensity control. In [13] a more precise technique is used based on inkjet cartridge technology. An ink cartridge has been modified to eject liquid scent rather than ink using the same principles of ink amount control, mixture and evaporation.

\section{Materials and Methods}

A digitally controlled scent element is presented to be used as off the shelf scent element for future olfactory displays fully assembled by the user. It can be controlled to generate a number of varying scent intensities to represent (with other scent elements) the content of the displayed information on the screen. It can be also used in other experimental of actual system such as in [14] a molecular communication system was created to send data via varying the delivery of isopropyl alcohol (rubbing alcohol) in to the air and receiving the dispersed molecules with alcohol sensor. A platform has been proposed to provide a non-expensive research tool for other fields of research. The scent element can be used in such platform reliably and efficiently and upgrading the research to have more than one material with non-overlapping detection characteristics by the sensor which can increase the data rate significantly. Table 1 shows a comparison between the proposed scent element and the performance and issues of other olfactory displays that are the most popular and recent in the literature.

Table 1. A Comparison Between the Proposed Scent Element and Popular Olfactory Displays

\begin{tabular}{|c|c|c|c|c|c|}
\hline $\begin{array}{c}\text { Reference } \\
\text { No. }\end{array}$ & Type & Speed & $\begin{array}{c}\text { Residual } \\
\text { scent } \\
\text { material }\end{array}$ & $\begin{array}{c}\text { Noise } \\
\text { level }\end{array}$ & Limitations \\
\hline$[1][2]$ & Air cannon & High & High & High & $\begin{array}{c}\text { Requires long time between ejections } \\
\text { to recover }\end{array}$ \\
\hline$[3-6]$ & $\begin{array}{c}\text { Solenoid valves and air } \\
\text { compressor }\end{array}$ & Medium & High & High & $\begin{array}{c}\text { Lab environment only, requires high } \\
\text { power and produces noise }\end{array}$ \\
\hline$[7][8]$ & $\begin{array}{c}\text { electro-osmotic micro } \\
\text { pump \& SAW atomizer }\end{array}$ & High & Low & Low & $\begin{array}{c}\text { Lab environment only, high voltage } \\
\text { for SAW device }\end{array}$ \\
\hline$[9]$ & $\begin{array}{c}\text { Solid scent material with } \\
\text { Peltier module }\end{array}$ & Low & Medium & $\begin{array}{c}\text { Very } \\
\text { low }\end{array}$ & $\begin{array}{c}\text { Heating takes very long time and } \\
\text { consumes high voltage 9v and } \\
\text { current } 1.2 \mathrm{~A}\end{array}$ \\
\hline
\end{tabular}




\begin{tabular}{|c|c|c|c|c|c|}
\hline$[10-12]$ & User worn with air pump & Medium & Medium & Low & Heavy equipment carried by the user \\
\hline$[13]$ & inkjet & High & Low & Low & $\begin{array}{c}\text { Heating the scented material causes } \\
\text { scent deformation }\end{array}$ \\
\hline Proposed & $\begin{array}{c}\text { Micro-porous Piezoelectric } \\
\text { film }\end{array}$ & High & Very low & $\begin{array}{c}\text { Very } \\
\text { low }\end{array}$ & No limitations found \\
\hline
\end{tabular}

\subsection{Scent Element Design}

The design of the scent element has several advantages such as compactness, spill proof easy to refill and printable by and 3D printer. It contains a reservoir for the liquid scented material (about 10cc) and a top opening to insert the high absorbent cotton core and a $2 \mathrm{~mm}$ edge to hold the piezoelectric micro porous film. The inside base of the scent element is taper shaped to collect all the scent material to bottom of the cotton core, the top contains a hole for refilling the scent element with proper scent material, see Figure 1 (a) and (b).

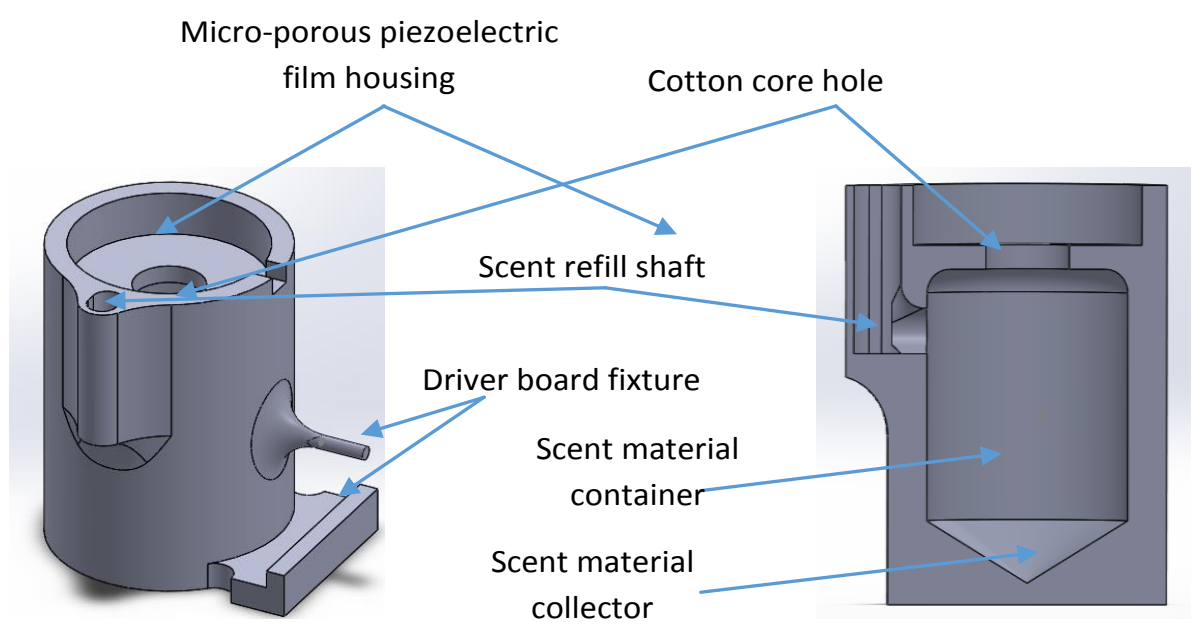

(a) External View

(b) Side Section View

\section{Figure 1. Scent Element Design View}

The described design represents the shell, chassis or housing of the scent element, other components are placed on this shell such as the circuit board providing the sufficient frequency to drive the ultrasonic piezoelectric film and the piezoelectric film itself using upper and lower silicone washers to hold it in place and providing seal and vibration freedom at the same time, these components are explained in details in the next sub sections.

\subsection{Piezoelectric Film}

The piezoelectric film used is a recently emerging type of films, it is called microporous piezoelectric film where the center of this film is a metal mesh with micro sized holes to extrude the scented material with micro sized droplets, see Figure 2 for the actual picture of the film. Placing the film directly above the cotton core will enable it to atomize the scent material absorbed by the cotton core by capillary action, the continuous vibration will atomize the material at the top and the cotton core will absorb more to complete the cycle, Figure 3 shows the scent element assembly in design and actual picture. 


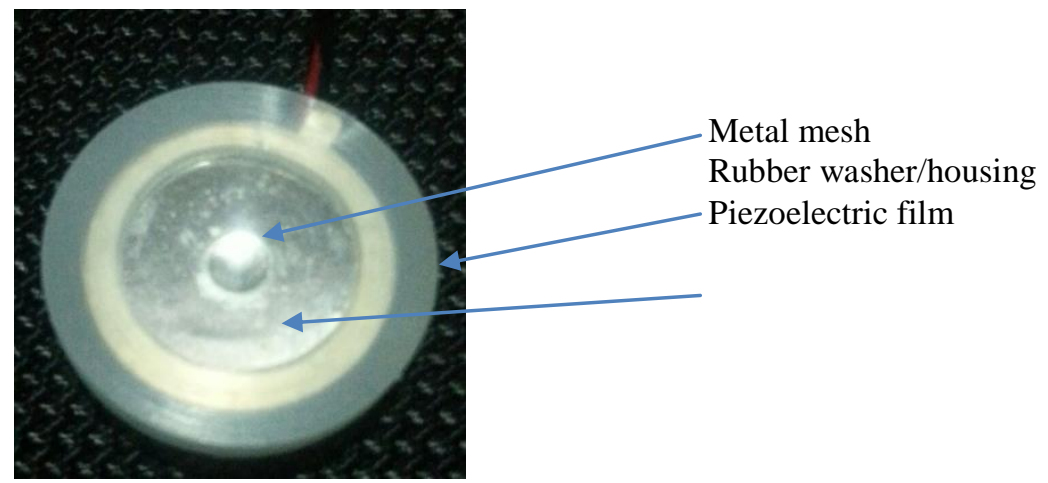

Figure 2. Actual Picture of Piezoelectric Film

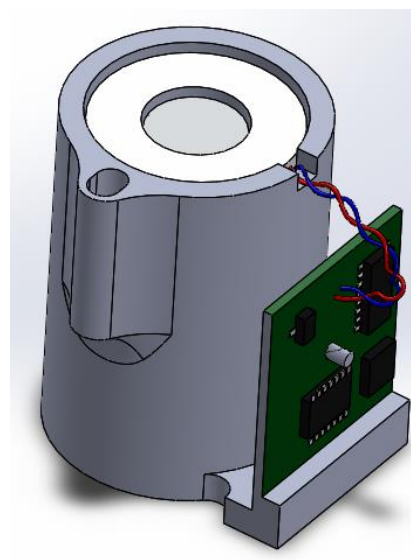

(a)

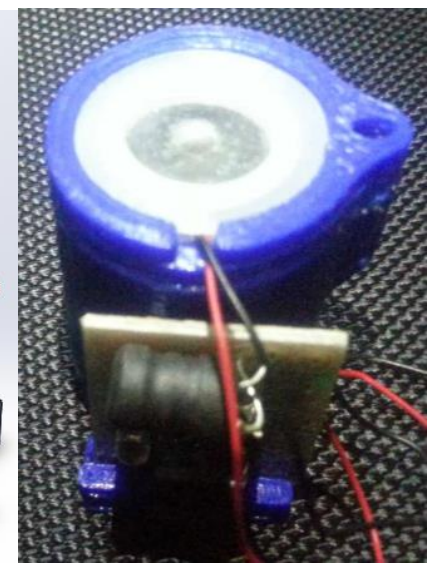

(b)

Figure 3. The Complete Scent Element (a) Design, (b) Actual Picture

The reason behind choosing the micro porous piezoelectric film is due to its versatility regarding voltage supply, size and other characteristics that illustrated as follows:

Properties of piezoelectric film

1. Diameter: $13.8 \mathrm{~mm}$.

2. Low driving voltage $: 3-12 \mathrm{~V}$.

3. High conversion efficiency, spray volume.

4. Exit aperture is very small $4 \mu \mathrm{m}$.

5. Frequency: $113 \mathrm{khz} \pm 5 \mathrm{khz}$.

6. Capacitance: $2700 \mathrm{PF} \pm 15 \%$.

7. Power: $1.5-2.0 \mathrm{~W}$.

8. Spray volume $30 \mathrm{ml} / \mathrm{h}$.

9. Can atomize essential oils, perfume, water based perfumes or even mixture of the mentioned materials.

10. life of more than 3000 hours.

\subsection{Speed}

Speed of delivery for the scent element is measured using a Tin Dioxide $\mathrm{SnO} 2$ sensor with aluminum ceramic tube AL2O3 which is highly responsive to alcohol placed $100 \mathrm{~cm}$ away from the scent element, the latter is filled with isopropyl alcohol (rubbing alcohol) diluted in water with $10 \%$ concentration, the distance is chosen to be the maximum distance to be set between a user and an interface device (peripheral device). In a controlled environment some experimental results were obtained regarding the response time and delivery, the ambient temperature was 23-25 degrees Celsius and humidity of 
$50-55 \mathrm{~g} / \mathrm{m} 3$, using $8.0 \mathrm{~cm} \times 7.5 \mathrm{~cm} 12 \mathrm{v}$ fan for wind generation, in initial tests a range of intensity values were sent consecutively to the scent element from the PC via a microcontroller and the response values of ppm (particles per million) were registered, Figure 4 shows the relation between the ejected alcohol and response ppm values by the sensor. When the microcontroller receives an ejection order from the PC it starts a timer and the response of the sensor stops that timer indicating detection has occurred.

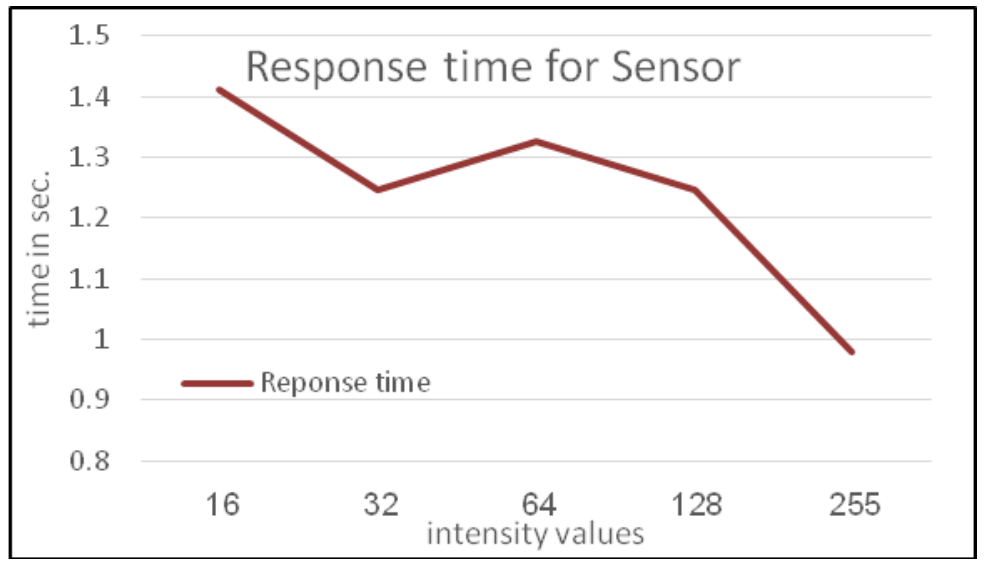

Figure 4. Sensor Response Times for Different Intensities

\subsection{Intensity}

The scent element is controlled using timed pulses, each pulse duration correspond to a different intensity of scent to be delivered to the user. By experimentation five distinctive levels of intensities can be produced by the scent element, the intensities ranges from 16 to 255 each of which can be represented by a byte of data sent to the microcontroller from the PC, being 16 as the lowest and 255 as the highest intensity. To measure these intensities a series of consecutive intensities are sent from the PC to the microcontroller from 16 to 255, measuring the response of the sensor for the same amount of alcohol to water dilution. Figure 5 shows the response values of the sensor with respect to the intensities delivered by the scent element.

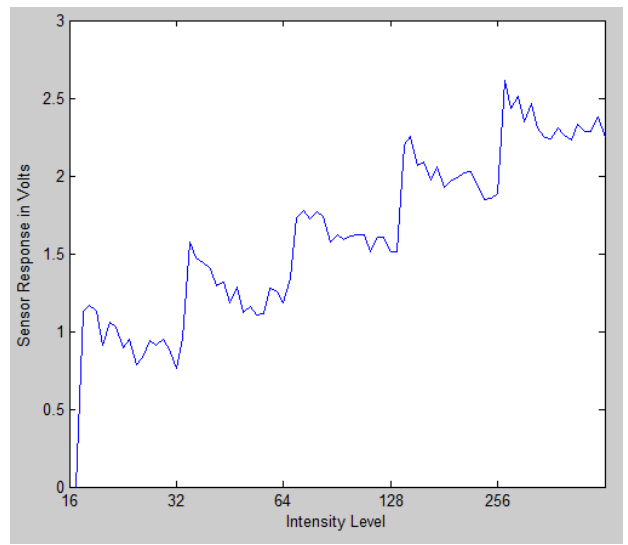

Figure 5. Intensity Levels and Sensor Response for each Intensity

\subsection{Durability}

The scent's element container is made from PLA plastic by 3D printing technology, it can be redesigned to have larger reservoir or larger piezoelectric film for applications requiring higher intensities of an olfactory display such as simulating fog particles, water splash or rain fall which requires higher volumes of water to be dispersed in the air in 
shorter time. The scent cartridge can be refilled easily by the upper refill hole after removing the cap and filling the desired scent.

\subsection{Scalability}

As mentioned earlier having the scent element to be such compact sized, it is possible to arrange more of these scent element into a small space having the advantage of size over the number of scent elements is decreased significantly, furthermore, the scent element has its own 3 wire connection 5V, GND and Pulse. The pulse is connected to a controlling microcontroller requiring on digital $\mathrm{I} / \mathrm{O}$ pin per scent element. The scent element dose not draw current from the microcontroller just needs a starting pulse to start activating and another pulse to declare the end of activation, to attach scent elements more than the actual number of digital I/O pins of the micro controller a shift register, a decoder or a de-multiplexer or any pin expansion approach, Figure 6 shows an olfactory display with $4 \times 5$ scent elements aligns as a matrix to provide scent material to the upper level of the olfactory display.

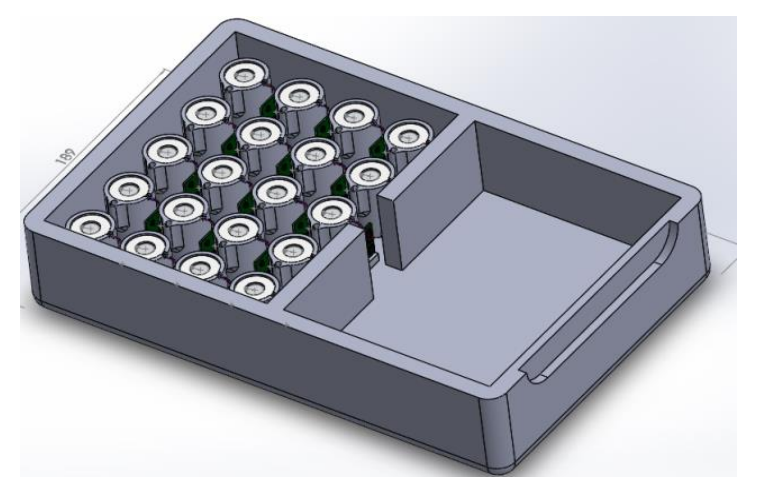
Figure 6. Olfactory Display with $4 \times 5$ Scent Element and Scalable by more
Scent Elements

\section{Conclusions}

Olfactory displays has gone through several phases of evolution, these phases treated the display as a whole device and expected to achieve the desired goals required directly from the display. Actually a bottom up redesign for the olfactory display has been done in this paper, creating a standalone modular scent element with the most recent and advanced diffusing micro-porous piezoelectric film to achieve best results with minimum impact in design and manufacturing. In the future a more compact and precise scent element can be built using a smaller sized micro-porous piezoelectric film to fit maybe a 10x10 scent diffusers in a 1 or 2 square centimeters area to be included in cellphones or any mobile devices without affecting the existing devices' design. Furthermore regarding the response time of the scent element it is very crucial to have a scent dispersing element that can provide the air born (gaseous) state of the formal liquid state in a very short time ensuring the delivery to the human's olfactory system, it is fair to mention that the air manipulation apparatus normally a fan or an air pump has a significant role in the operation of delivery especially the travelling speed of the scent from the ejection point of the olfactory display to the reception point of the human's olfactory system. Also the proposed scent element ensures minimum residual scent in the olfactory display due to precise atomization method which is considered a major inherited problem in olfactory displays. A numerous kinds of scent material handling is also one of the challenges regarding scalability, until now there is no 
ability to mix the raw ingredients of a scent on demand such apparatus is either impractical to implement or too complex to be considered as a desktop end user's equipment so the direction is toward ready scent ejection which dictates the problem of having each scent type to have its own scent element and this was solved by the proposed scent element with the flexibility of scalability.

\section{Acknowledgment}

This work was partially supported by the National Natural Science Foundation of China (Grant No. 61173107), the National High Technology Research and Development Program of China (Grant No. 2012AA01A301-01), the Special Project on the Integration of Industry, Education and Research of Guangdong Province, China (Grant No. 2011A091000027) and the Project on the Integration of Industry, Education and Research of Huizhou, Guangdong Province, China (Grant No. 2012C050012012).

\section{References}

[1] Y. Yanagida, S. Kawato, H. Noma, A. Tomono and N. Tesutani, "Projection based olfactory display with nose tracking", Proceedings. IEEE Virtual Reality, March (2004), pp.43-50.

[2] Y. Yanagida, T. Tanakamaru, H. Nagayanagi, Y. Nomura and T. Aritake, "Flat-shaped, front-face-drive scent projector", Virtual Reality Short Papers and Posters (VRW), IEEE, Costa Mesa, CA, March (2012), pp.159-160.

[3] T. Nakamoto, K. Hashimoto, T. Aizawa and Y. Ariyakul, "Multi-component olfactory display with a SAW atomizer and micropumps controlled by a tablet PC", IEEE International Frequency Control Symposium (FCS), Taipei, May (2014), pp.1-4.

[4] T. Nakamoto, M. Kinoshita, K. Murakami and A. Yossiri, "Demonstration of Improved Olfactory Display using Rapidly-Switching Solenoid Valves", IEEE Virtual Reality Conference, VR, Lafayette, LA, March (2009), pp.301-302.

[5] T. Nakamoto and H. P. D. Minh, "Improvement of olfactory display using solenoid valves", IEEE Virtual Reality Conference, VR, Charlotte, NC, March (2007), pp.179-186.

[6] T. Nakamoto and K. Yoshikawa, "Movie with Scents Generated by Olfactory Display Using Solenoid Valves", IEEE Virtual Reality Conference, March (2006), pp.291-292.

[7] Y. Ariyakul and T. Nakamoto, "Olfactory display using a miniaturized pump and a SAW atomizer for presenting low-volatile scents", IEEE Virtual Reality Conference, VR, Singapore, March (2011), pp.193-194.

[8] Y. Ariyakul, Y. Hosoda and T. Nakamoto, "Improvement of odor blender using electroosmotic pumps and SAW atomizer for low-volatile scents", IEEE Sensors, Taipei, October (2012), pp.1-4.

[9] D. W. Kim, H. C. Yeong, K. Nishimoto, Y. Kawakami, S. Kunifuji and H. Ando, "Development of Aroma Card Based Soundless Olfactory Display", 16th IEEE International Conference on Electronics, Circuits, and Systems, Yasmine Hammamet, Tunesia, December (2009), pp.703-706.

[10] T. Yamada,S. Yokoyama, T. Tanikawa, K. Hirota and M. Hirose, "Wearable Olfactory Display: Using Odor in Outdoor Environment", IEEE Virtual Reality Conference, March (2006), pp.199-206.

[11] T. Narumi, T. Kajinami, S. Nishizaka, T. Tanikawa and M. Hirose, Pseudo-gustatory display system based on cross-modal integration of vision, olfaction and gustation, IEEE Virtual Reality Conference, VR, Singapore, March (2011), pp.127-130.

[12] K. Ohtsu, J, Sato, Y. Bannai and K. Okada, Scent Presentation Technique of Pulse Ejection Synchronized with Breathing, Ninth Annual International Symposium on Applications and the Internet, SAINT '09, Bellevue, WA, July (2009), pp.125-128.

[13] A. Kadowaki, D. Noguchi, S. Sugimoto, Y. Bannai and K. Okada, "Development of a High Performance Olfactory Display and Measurement of Olfactory Characteristics for Pulse Ejections", 10th IEEE/IPSJ International Symposium on Applications and the Internet (SAINT), Seoul, July (2010), pp.1-6.

[14] N. Farsad, W. Guo and A. W. Eckford, "Tabletop Molecular Communication: Text Messages through Chemical Signals", PLoS One, vol.8, no.12, (2013), pp.1-13. 


\section{Authors}

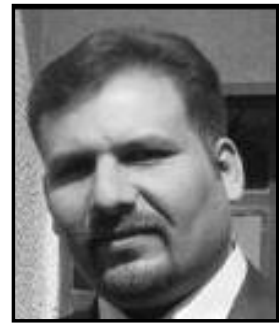

Saad Hameed Abid was born in Baghdad - Iraq in 7 June 1979, he received his B.Sc. Degree in computer science at AL-Mansour University College 2001, and Masters Degree in computer Sciences, Iraqi committee for computer and informatics in 2004, he continued working in academic teaching in AL-Mansour University College for 11 years, through that time all of his research was self-funded and concentrated in automation and control, he has been promoted from assistant Lecturer to Lecturer in 2011, he is now a Ph.D. degree student at Hunan University in P.R. China.

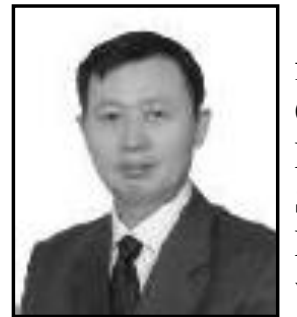

Zhiyong Li received the MSc degree in System Engineering from National University of Defense Technology, Changsha, China, in 1996 and PhD degree in Control Theory and Control Engineering from Hunan University, Changsha, China, in 2004. Since 2004, he joined the College of Information Science and Engineering of Hunan University. Now, he is a Full Professor with Hunan University, member of China Computer Federation $(\mathrm{CCF})$. His research interests include visual object tracking, embedded computing system, dynamic multi-objective optimization evolutionary algorithm and tasks scheduling optimization in cloud computing. He has published more than 50 papers in international and domestic journals and conferences.

Prof. Li obtained several awards from academic organizations and conferences, such as the Champion of the Future Challenge: Intelligent Vehicles and Beyond, FC'09, which was hosted by the National Natural Science Fund Committee of China in 2009.

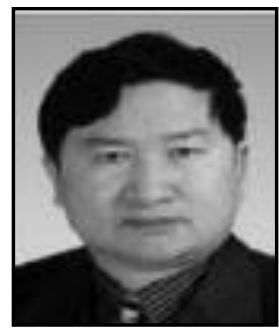

Renfa Li was born in Hunan - China in 1957, he works as Professor and $\mathrm{PhD}$ supervisor of Hunan University, Senior member of China Computer Federation (CCF), his main research interests include embedded system, artificial intelligence and optimization theory etc.

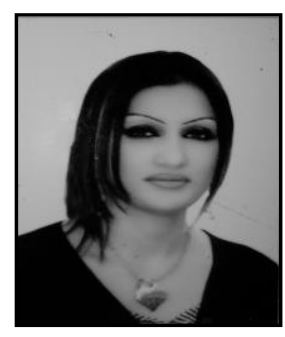

Jumana Waleed is a Ph.D. student in the School of information science and Engineering at Central South University, Changsha, China. Her research activity focuses on image processing, and information security working on digital watermarking. She received the B.S. degree in computers sciences from the Al-Yarmouk University College, Iraq, in 2004, and the M.S. degree in Computer Science/Data Security from the University of Technology, Baghdad, Iraq, in 2009. 